TITLE:

\title{
Emission characteristics of Spindt- type platinum field emitters improved by operation in carbon monoxide ambient
}

\section{$\operatorname{AUTHOR}(\mathrm{S}):$}

Gotoh, Y; Nozaki, D; Tsuji, H; Ishikawa, J; Nakatani, T; Sakashita, T; Betsui, K

\section{CITATION:}

Gotoh, Y ...[et al]. Emission characteristics of Spindt-type platinum field emitters improved by operation in carbon monoxide ambient. JOURNAL OF VACUUM SCIENCE \&

TECHNOLOGY B 2001, 19(3): 912-915

\section{ISSUE DATE:}

2001-05

URL:

http://hdl.handle.net/2433/39777

RIGHT:

Copyright 2001 American Vacuum Society 


\title{
Emission characteristics of Spindt-type platinum field emitters improved by operation in carbon monoxide ambient
}

\author{
Y. Gotoh, ${ }^{\text {a) }}$ D. Nozaki, H. Tsuji, and J. Ishikawa \\ Department of Electronic Science and Engineering, Kyoto University, Yoshida-honmachi, Sakyo-ku, \\ Kyoto 606-8501, Japan \\ T. Nakatani, T. Sakashita, and K. Betsui \\ Fujitsu Laboratories, Ltd., 64 Nishiwaki, Ohkubo-cho, Akashi, Hyogo 674-8555, Japan
}

(Received 20 September 2000; accepted 10 April 2001)

\begin{abstract}
The electron emission characteristics of Spindt-type platinum field emitters that were improved by operation in a carbon monoxide gas ambient were investigated in detail. We present differences in the emission characteristics, such as the change of Fowler-Nordheim characteristics in the S-K chart, change in current fluctuation and emission pattern, and resistance against oxygen exposure due to the improvement. We found that the work function increased slightly during the introduction of carbon monoxide gas up to $10^{-4} \mathrm{~Pa}$; but when the gas was evacuated, the work function recovered. The work function finally decreased at a pressure of $10^{-3} \mathrm{~Pa}$. The emission characteristics were degraded during the oxygen exposure both for the nontreated and treated emitters. However, degradation was less significant for the carbon-monoxide-treated emitter than for the nontreated emitter. All these results suggest that the improvement can be attributed to the presence of carbon atoms or a carbon layer at the surface of the emitter. (C) 2001 American Vacuum Society. [DOI: 10.1116/1.1376392]
\end{abstract}

\section{INTRODUCTION}

To develop a miniaturized cathode that exhibits a stable current and long lifetime, one must find a material that has superior properties for field emission in a poor vacuum. We have investigated various kinds of materials, including transition-metal nitrides ${ }^{1}$ and diamonds, ${ }^{2}$ but elemental metals are potential materials for these cathodes because of ease of processing. We already reported on the electron emission characteristics for some Spindt-type cathodes with various kinds of elemental metal emitters, finding that a platinum (Pt) emitter was most stable because of its inertness. ${ }^{3}$ However, we also found that even for the Pt emitters, the emission property was strongly affected by the ambient gas, especially for oxygen $\left(\mathrm{O}_{2}\right),{ }^{4}$ which was also reported by Chalamara et al. ${ }^{5}$

One of the easiest ways to improve the above property is to modify the emitter surface by a post-treatment. It is known that hydrogen or helium gas introduction improves the emission property of Spindt-type molybdenum (Mo) emitters. ${ }^{6,7}$ However, these improvements do not continue for a long time because of the desorption of the adsorbed gas molecules. Itoh $\mathrm{et} \mathrm{al.} .^{8}$ reported on the improvement of the emission property by operation in a methane $\left(\mathrm{CH}_{4}\right)$ or carbon monoxide (CO) ambient. Matsukawa et al. ${ }^{9}$ also reported the improved performance of silicon emitter arrays by operation in an ethylene $\left(\mathrm{C}_{2} \mathrm{H}_{4}\right)$ ambient.

In a similar manner, we have successfully improved the emission characteristics of Spindt-type Pt field emitters by operation in a $\mathrm{CO}$ ambient. ${ }^{10}$ As a result, the driving voltage was significantly reduced as compared with that before the

\footnotetext{
a) Author to whom correspondence should be addressed; electronic mail: ygotoh@kuee.kyoto-u.ac.jp
}

treatment. In this article, we examine the electron emission characteristics of these improved emitters, especially the resistance against $\mathrm{O}_{2}$ exposure of these improved emitters, because this issue is critical for the development of field emission displays. ${ }^{4,5}$ The response against $\mathrm{O}_{2}$ introduction also gives us a hint concerning the reaction occurring at the emitter surface.

\section{EXPERIMENTAL PROCEDURE}

\section{A. Fabrication of platinum emitters}

The Spindt-type Pt field emitters were fabricated by our process ${ }^{11}$ based on the Spindt process. ${ }^{12}$ Details of the fabrication are given elsewhere. ${ }^{3}$ Because the Pt emitter was thin, metal materials that had a larger cone-half angle were deposited prior to deposition $\mathrm{Pt}$, in order to adjust the height of the emitter to be the same as the upper surface of the gate electrode. In this experiment, one-tip emitters were used because the use of an array makes it difficult to interpret the results.

\section{B. Measurement system}

The fabricated device was mounted on a TO-5 heading, and its fundamental emission characteristics were examined. Details of the measurement system are provided elsewhere. ${ }^{4}$ An external collector, which was a phosphor-coated conductive glass plate, was placed about $5 \mathrm{~mm}$ above the device. The gate electrode was grounded, and the emitter was negatively biased $\left(V_{\mathrm{EG}}\right)$. The collector was biased to positive 200 $\mathrm{V}$. The current $\left(I_{\mathrm{C}}\right)$ and emission pattern on this collector were investigated. The system was pumped down by a turbomolecular pump and a sputter-ion pump. The residual gas pressure was about $10^{-7} \mathrm{~Pa}$. The $\mathrm{CO}$ and $\mathrm{O}_{2}$ gases were introduced through a variable leak valve. 


\section{Improvement of platinum emitter with carbon monoxide gas}

The electron emission characteristics were improved by introducing $\mathrm{CO}$ gas during the operation of the emitter. ${ }^{10}$ The improvement described elsewhere ${ }^{10}$ was observed for eight emitters with sufficient reproducibility. Here, we describe the process very briefly. First, the emitter was aged for several hours up to an emission current of about $20 \mu \mathrm{A}$. After the aging, we gradually introduced $\mathrm{CO}$ gas. The emission current was adjusted to $1 \mu \mathrm{A}$, and we then introduced $\mathrm{CO}$ gas to $10^{-5} \mathrm{~Pa}$. Keeping the applied voltage constant, the variation of the current was monitored. Current-voltage characteristics in the $\mathrm{CO}$ ambient were also measured for some emitters. After about 30 min exposure, the $\mathrm{CO}$ gas was evacuated, and current-voltage characteristics were measured. We then introduced $\mathrm{CO}$ gas to $10^{-4} \mathrm{~Pa}$ without changing the applied voltage. We repeated this process until the pressure came to $10^{-3} \mathrm{~Pa}$. At a pressure of $10^{-3} \mathrm{~Pa}$, the current began to increase, and we stopped introducing gas when the current exceeded the initial value of $1 \mu \mathrm{A}$ (mostly at several microamps). We call the above process "CO treatment," and an emitter treated by this process is designated as a "CO-treated emitter." Also an emitter without the treatment is designated as " nontreated emitter." We did not see any improvement by introducing $\mathrm{CO}$ gas without activating the device.

\section{Introduction of oxygen gas}

Introduction of $\mathrm{O}_{2}$ gas to investigate the resistance against $\mathrm{O}_{2}$ exposure was performed in a similar manner as the $\mathrm{CO}$ gas introduction. During the $\mathrm{O}_{2}$ introduction, the emitter was activated.

\section{E. Evaluation of emission characteristics}

The evaluation of the emission characteristics was performed by the $\mathrm{S}-\mathrm{K}$ chart. ${ }^{13-15}$ The $\mathrm{S}-\mathrm{K}$ chart is a diagram in which the abscissa and ordinate are the intercept and slope, respectively, of the Fowler-Nordheim $(\mathrm{F}-\mathrm{N})$ plot, and it provides us with useful information on the apex radius and work function of the emitter. Emission patterns and current fluctuations were also considered.

\section{ELECTRON EMISSION CHARACTERISTICS}

\section{A. Change in current-voltage characteristics}

Figure 1 shows the typical Fowler-Nordheim plots of the current-voltage characteristics before and after exposure to $\mathrm{CO}$ gas. The open triangles and open circles show the emission characteristics of virgin emitter and after exposure to $10^{-3} \mathrm{~Pa}$, respectively. After the improvement, the current increased by two orders of magnitude. The driving voltage was reduced by up to $20 \mathrm{~V}$.

To investigate the mechanism of the improvement, we analyzed in detail the emission characteristics during $\mathrm{CO}$ introduction. Figure 2 shows the change of the $\mathrm{F}-\mathrm{N}$ characteristics in the $\mathrm{S}-\mathrm{K}$ chart. The solid circle shows the characteristic in ultrahigh vacuum (UHV) just after the aging. The

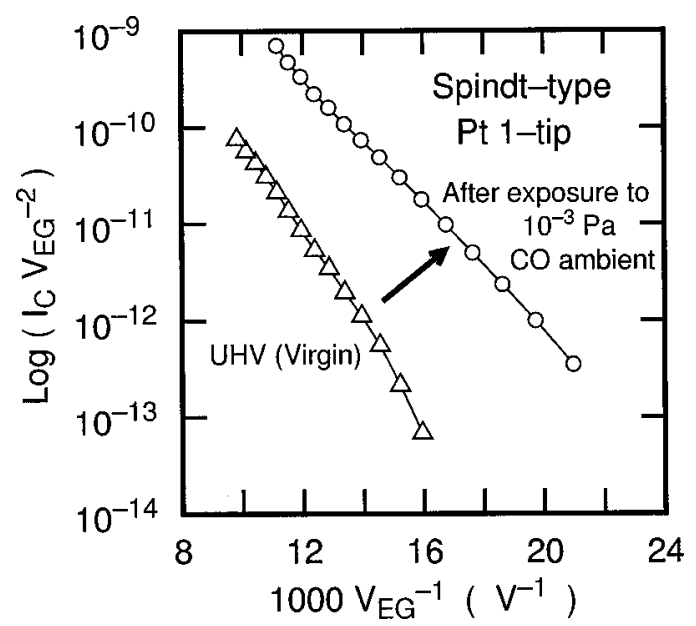

FIG. 1. Fowler-Nordheim plots of the emission characteristics before and after exposure to carbon monoxide gas.

open and closed symbols indicate the characteristics during $\mathrm{CO}$ exposure and in UHV after $\mathrm{CO}$ exposure, respectively.

The square, rhombus, and triangle symbols indicate the CO gas pressure of $10^{-5}, 10^{-4}$, and $10^{-3} \mathrm{~Pa}$, respectively. The arrows show the order of the variation. Dashed lines indicate the equiwork functions (EWFs) that were suggested by the variation of the characteristics of the emitter in the UHV environment. ${ }^{10,15}$ Because it is difficult to determine the absolute value of the work function, these lines are drawn with an arbitrary interval. The number shown next to the EWF line indicates the approximate driving voltage for extraction of $40 \mathrm{nA}$. The upper right direction with respect to the EWF line indicates a decrease of the work function and the lower left direction indicates an increase of the work function. From the result, we see that the work function increased slightly due to $\mathrm{CO}$ introduction at the lower gas pressure.

However, when the $\mathrm{CO}$ gas was evacuated, the work function seems to recover. With an introduction of $10^{-3} \mathrm{~Pa}$, the work function began to decrease.

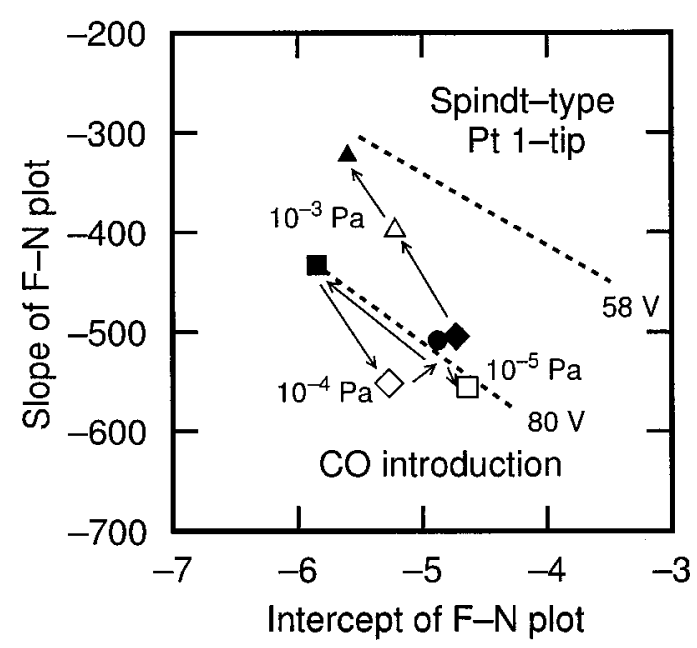

FIG. 2. Change of electron emission characteristics in the $\mathrm{S}-\mathrm{K}$ chart. 


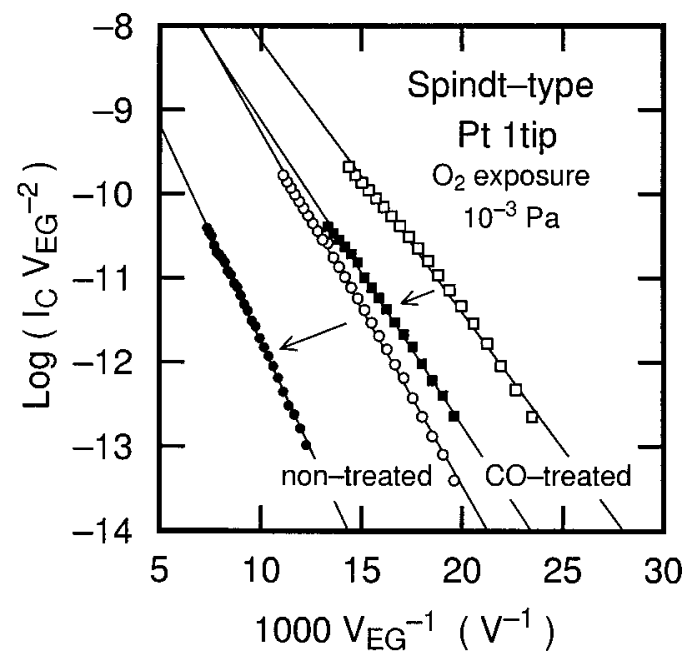

FIG. 3. Fowler-Nordheim plots of the emission characteristics before and after exposure to oxygen ambient for the nontreated and $\mathrm{CO}$-treated emitters.

\section{B. Current fluctuation}

Improvement of the emission characteristics also improved the current fluctuation of the emitter. Fourier analysis revealed that the current fluctuation in UHV operation was lowered by one order of magnitude after the $\mathrm{CO}$ treatment. This reduction agrees with the empirical relation between work function and emission stability. ${ }^{13,16}$

\section{Change in emission pattern}

The virgin Pt emitters showed alternative emission from multiple sites during operation in UHV. After the CO treatment, the emission pattern was fixed and did not change significantly. A spot was seen at the center, and two bright arcs were seen above and below the center spot, implying that the emission site was almost fixed by the introduction of CO gas.

\section{Resistance against exposure to oxygen gas}

The changes in electron emission characteristics when the cathode is subject to an oxygen ambient were compared between the nontreated and CO-treated emitters. The emission current first decreased rapidly and then the rate of decrease became smaller for both emitters. The final current in $\mathrm{O}_{2}$ ambient was about two orders of magnitude lower than that in UHV. After exposure to $10^{-3} \mathrm{~Pa}$, the $\mathrm{O}_{2}$ gas was evacuated and the emission characteristics of the emitter were examined in an UHV environment.

Figure 3 shows the change in Fowler-Nordheim plots before and after the $\mathrm{O}_{2}$ introduction, for the nontreated and CO-treated emitters. The open and solid symbols indicate before and after the $\mathrm{O}_{2}$ introduction, and the circle and square symbols indicate the characteristics for the nontreated and CO-treated emitters, respectively. The emission characteristics degraded for both the nontreated and CO-treated emitters. However, the change in the characteristics was smaller for the CO-treated emitter.
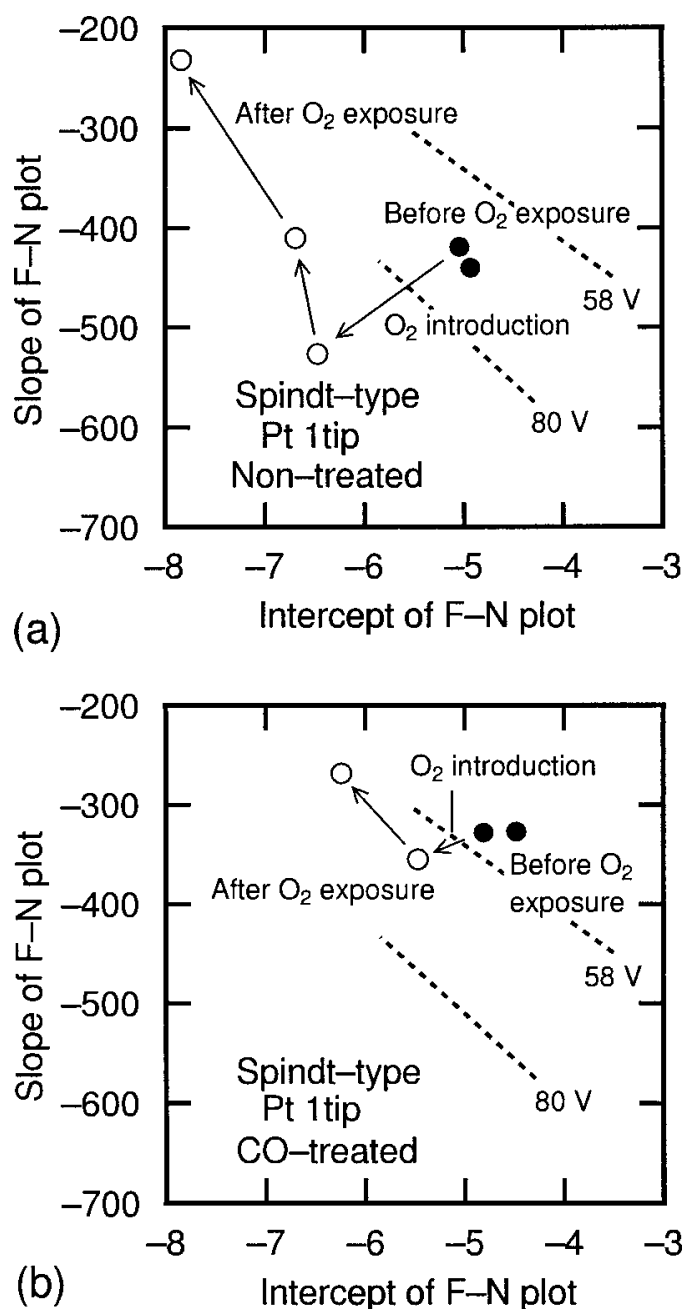

FIG. 4. Change of F-N characteristics in the $\mathrm{S}-\mathrm{K}$ chart: (a) nontreated $\mathrm{Pt}$ emitter and (b) CO-treated Pt emitter.

Figures 4(a) and 4(b) show the change of the emission characteristics in the $\mathrm{S}-\mathrm{K}$ chart through the operation in $\mathrm{O}_{2}$ ambient for the nontreated and CO-treated emitters, respectively. In these figures, dashed lines indicate the EWF lines. For the nontreated emitter, the shift due to $\mathrm{O}_{2}$ exposure is larger, whereas the shift is smaller for the CO-treated emitter. From these results, the resistance against $\mathrm{O}_{2}$ introduction was improved. This figure also shows the emission characteristics at a long time after the evacuation of $\mathrm{O}_{2}$ gas. The final characteristics in Figs. 4(a) and 4(b) are those after 3 and $14 \mathrm{~h}$, respectively. The position of the $\mathrm{F}-\mathrm{N}$ characteristics in the $\mathrm{S}-\mathrm{K}$ chart came onto the extrapolated position of the EWF line before $\mathrm{O}_{2}$ exposure. This result means that the emission characteristics gradually recovered, but have a smaller emission area.

\section{DISCUSSION}

As we already discussed, the improvement of the emission properties are due to the reduction of the effective work function of the emitter. ${ }^{10}$ This conclusion is also supported by the fact of the reduction of the current fluctuation associated with the reduction of the work function. The suggested 
processes that reduce the work function due to the $\mathrm{CO}$ treatment are: (a) reduction of the emitter surface due to a catalytic reaction between $\mathrm{O}$ and $\mathrm{CO}$ (oxidation of $\mathrm{CO}$ ), and (b) attachment of $\mathrm{C}$ atoms onto the $\mathrm{Pt}$ emitter surface. If the reduction process is a major reason, the tarnished layer of the emitter surface would be removed by the $\mathrm{CO}$ treatment. In this case, the surface would become sensitive to the adsorption of $\mathrm{O}_{2}$ molecules compared to a dirty surface, i.e., the nontreated emitter. However, experiments showed greater resistance against the $\mathrm{O}_{2}$ gas for the CO-treated emitter. Furthermore, a clean surface may exhibit an occasional change in the emission pattern due to adsorption of residual gas molecules, but we did not see such changes. This suggest that the reduction process is not the major reason. The latter case is such that the dissociation of $\mathrm{CO}$ molecules at the surface occurs or electron-impact dissociation of $\mathrm{CO}$ molecules results in the deposition of $\mathrm{C}$ atoms. As for the work function of the Pt surface with the presence of C layers, some researchers ${ }^{17,18}$ reported the reduction of the work function up to $1.5 \mathrm{eV}$. Thus, the presence of $\mathrm{C}$ atoms or a $\mathrm{C}$ layer may be a plausible reason for the reduction of work function. Further investigation by transmission electron microscopy will be necessary to confirm the earlier hypothesis.

\section{CONCLUSION}

The electron emission characteristics of CO-treated emitters were investigated. The CO-treated emitter showed greater resistance against the exposure to $\mathrm{O}_{2}$ gas, as compared with the nontreated emitter. The improved emitter exhibited low current fluctuation and stable emission pattern. These improvements are due to a reduction in the work function. The reduction of the work function may be attributed to the presence of $\mathrm{C}$ atoms or a $\mathrm{C}$ layer on the Pt surface. This treatment, together with the use of platinum as a cathode material, will realize stable and long lifetime field emission arrays in the near future.

${ }^{1}$ M. Nagao, Y. Gotoh, T. Ura, H. Tsuji, and J. Ishikawa, J. Vac. Sci. Technol. B 17, 623 (1999).

${ }^{2}$ Y. Gotoh, T. Kondo, M. Nagao, H. Tsuji, J. Ishikawa, K. Hayashi, and K. Kobashi, J. Vac. Sci. Technol. B 18, 1018 (2000).

${ }^{3}$ M. Nagao, K. Utsumi, Y. Gotoh, H. Tsuji, J. Ishikawa, T. Nakatani, T. Sakashita, and K. Betsui, Appl. Surf. Sci. 146, 182 (1999).

${ }^{4}$ Y. Gotoh, K. Utsumi, M. Nagao, H. Tsuji, J. Ishikawa, T. Nakatani, T. Sakashita, and K. Betsui, J. Vac. Sci. Technol. B 17, 604 (1999).

${ }^{5}$ B. R. Chalamara, R. M. Wallance, and B. E. Gnade, J. Vac. Sci. Technol. B 16, 2859 (1998).

${ }^{6}$ I. Brodie and C. A. Spindt, Vacuum Microelectronics, Advances in Electronics and Electron Physics Vol. 83 (Academic, Orlando, 1992).

${ }^{7}$ B. R. Chalamara, R. M. Wallance, and B. E. Gnade, J. Vac. Sci. Technol. B 16, 2855 (1998).

${ }^{8}$ S. Itoh, T. Niiyama, and M. Yokoyama, J. Vac. Sci. Technol. B 11, 647 (1993).

${ }^{9}$ T. Matsukawa, S. Kanemaru, K. Tokunaga, and J. Itoh, J. Vac. Sci. Technol. B 18, 952 (2000).

${ }^{10}$ Y. Gotoh, D. Nozaki, H. Tsuji, J. Ishikawa, T. Nakatani, T. Sakashita, and K. Betsui, Appl. Phys. Lett. 77, 588 (2000).

${ }^{11}$ T. Nakatani, T. Sakashita, O. Toyoda, K. Inoue, T. Kosaka, N. Kondo, S. Fukuta, and K. Betsui, Proc. 3rd Intl. Display Workshops, Kobe, 1996, p. 127.

${ }^{12}$ C. A. Spindt, I. Brodie, L. Humphrey, and E. R. Westerberg, J. Appl. Phys. 47, 5248 (1976).

${ }^{13}$ Y. Gotoh, M. Nagao, M. Matsubara, K. Inoue, H. Tsuji, and J. Ishikawa, Jpn. J. Appl. Phys., Part 2 35, L1297 (1996).

${ }^{14}$ J. Ishikawa, H. Tsuji, Y. Gotoh, T. Sasaki, T. Kaneko, M. Nagao, and K. Inoue, J. Vac. Sci. Technol. B 11, 403 (1993).

${ }^{15}$ Y. Gotoh, H. Tsuji, and J. Ishikawa, Appl. Surf. Sci. (submitted).

${ }^{16}$ Y. Gotoh, H. Tsuji, and J. Ishikawa, J. Vac. Sci. Technol. B, these proceedings.

${ }^{17}$ X. Q. D. Li, T. Radojicic and R. Vanselow, Surf. Sci. 225, L29 (1990).

${ }^{18}$ I. D. Hughes and H. M. Montagu-Pollack, J. Phys. D 3, 228 (1970). 